\title{
Hyperspectral imaging of gold dimers
}

\author{
Maxim Bashevoy, Fredrik Jonsson and Nikolay I. Zheludev \\ Optoelectronics Research Centre, University of Southampton, SO17 1BJ, United Kingdom \\ F. Javier García de Abajo \\ Instituto de Óptica, CSIC, Serrano 121, 28006 Madrid, Spain \\ Isabel Pastoriza-Santos and Luis M. Liz Marzán

\section{Departamento de Química Física and Unidad Asociada CSIC-Universidade de Vigo, 36310 Vigo, Spain}

We report on the first realization of a hyperspectral imaging (HSI) technique of surface plasmon polaritons excited in metallic nanostructures by a scanning electron beam. The HSI image is formed by scanning a plasmonic structure with a focused electron beam, simultaneously recording the spectra of light emission from decoupled plasmons by a spectrum analyzer. By scanning the sample in this manner, one obtains a data cube consisting of the spatial coordinates of the position of the injected electron beam and the corresponding plasmon emission spectra.

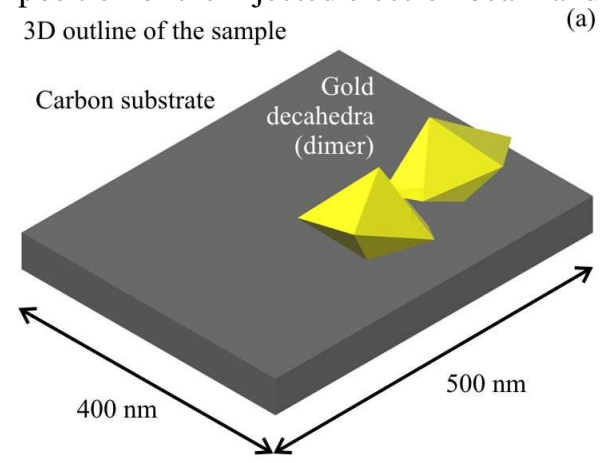

Plasmon emission at $550 \mathrm{~nm}$

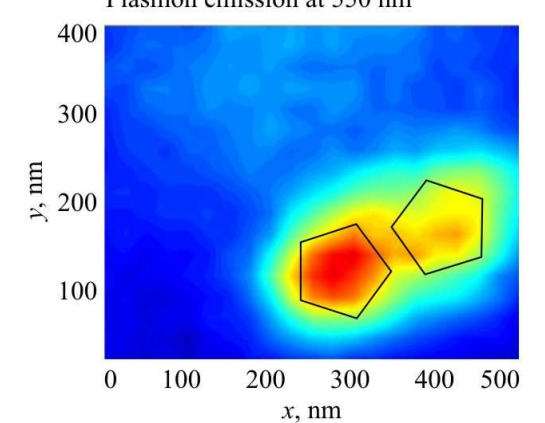

(b)

Plasmon emission at $650 \mathrm{~nm}$

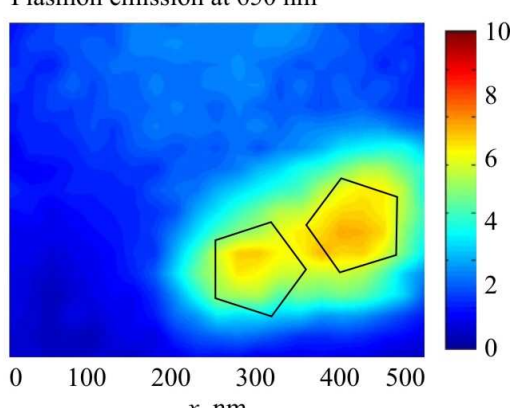

Fig. 1. Electron beam excitation of plasmons in the dimer (a), consisting of two $100 \mathrm{~nm}$ gold decahedra separated by a $20 \mathrm{~nm}$ gap. An asymmetric mode of excitation is found at $550 \mathrm{~nm}$ (b) while a symmetric mode is found at $650 \mathrm{~nm}(\mathrm{c})$.

We performed plasmonic HSI on a dimer [1] consisting of two gold $100 \mathrm{~nm}$ decahedra on a carbon substrate (Fig. 1). For the dimer nanostructure we demonstrate its imaging as a plasmonic nanostructure, visualizing its plasmonic modes, and we identify the electron beam excitation of symmetric, asymmetric and hybridization modes (Fig. 2).

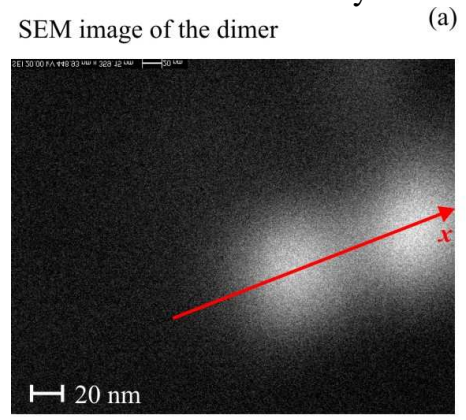

$\rightarrow$ Cross-section axis $x$

(a)

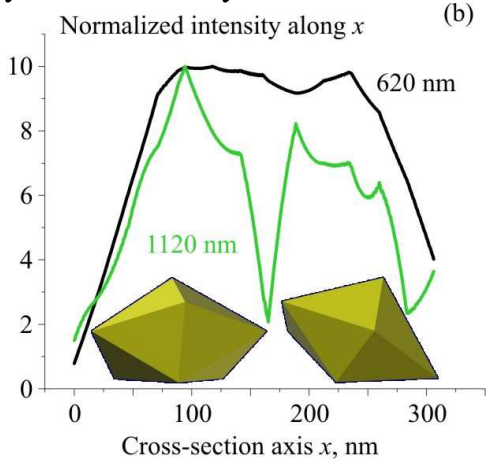

Fig. 2. Intensity distribution along the axis of the dimer (a), illustrating the hybridization mode at $620 \mathrm{~nm}$, at which the nanoparticles collectively act as a dipole, and the separated mode at $1120 \mathrm{~nm}$, in which they act as separate entities (b).

In conclusion, the demonstrated technique can be used in quick examination of plasmonic nanostructures with the resolution of the scanning electron microscope in a single scan. It has all the advantages of plasmon generation by injection of a free electron beam [2,3], such as fast repositioning on the nanostructure and a high localization and intensity of the plasmon source.

\section{References}

1. I. Romero, J. Aizpurua, G. W. Bryant, F. J. García de Abajo, "Plasmons in nearly touching metallic nanoparticles: singular response in the limit of touching dimers," Optics Express 14, 9988 (2006).

2. M. V. Bashevoy, F. Jonsson, A. V. Krasavin, N. I. Zheludev, Y. Chen, and M. I. Stockman, "Generation of Traveling Surface Plasmon Waves by Free-Electron Impact," Nano Letters 6, 1113 (2006).

3. J. T. van Wijngaarden, E. Verhagen, A. Polman, C. E. Ross, H. J. Lezec, and H. A. Atwater, "Direct imaging of propagation and damping of nearresonance surface plasmon polaritons using cathodoluminescence spectroscopy," Appl. Phys. Lett. 88, 221111 (2006). 